\title{
CONSTRUCTING AND ROLLING OUT THE NEW SOUTH AFRICAN SIGN LANGUAGE (SASL) CURRICULUM - REFLEXIVE CRITIQUE
}

Ruth Morgan, University of the Witwatersrand

Meryl Glaser, University of the Witwatersrand

Lucas Magongwa, University of the Witwatersrand

South Africa has one of the most progressive constitutions in the world protecting the linguistic and cultural rights of Deaf people. However, there is a huge gap between policies and reality in schools for Deaf learners in South Africa. In this paper we critically unpack and reflexively explore the linguistic and cultural issues that emerged during the process of creating and implementing the new South African Sign Language (SASL) curriculum. This curriculum was produced as a result of a landmark court case. We use an ethnographic framework based on our own reflections, discussions with Deaf people, notes from meetings, and discussions with the other team members. Why and how did the process that started off with so much energy, excitement and goodwill break down? We argue that a key reason for this breakdown is inadequate linguistic knowledge and cultural sensitivity in relation to SASL and Deaf cultural identity. The paper concludes with an outline of lessons learnt.

\section{INTRODUCTION}

In this paper we critically and reflexively deliberate on the process of creating and implementing the new South African Sign Language (SASL) curriculum. We decided to write this paper in order to reflect on what we did, the decisions we took individually and collaboratively and to assess why in our view the process broke down due to structural limitations (Archer, 2007; Brew et al., 2013). The curriculum was produced as a result of a landmark court case that we discuss below. We consider why there were serious problems in the early stages of implementation (teacher training and materials development). Why and how did the process that started off with so much energy, excitement and goodwill break down? We will argue that a key reason for this breakdown was that at the end of 2014 key people in the Department of Education who controlled this process refused to adequately acknowledge the role of linguistic knowledge and cultural sensitivity in relation to SASL and Deaf cultural identity. The Deaf ${ }^{i}$ community was so dissatisfied that the Deaf Federation of South Africa (DeafSA) considered taking the Department of Basic Education back to court.

We first provide the legal and socio-political context that forms the backdrop for the 2009 court case and outline the struggle for linguistic rights for Deaf learners during the first 15 years of schooling after the advent of democracy. South Africa has a complex history of colonial immigration and rule; local turmoil for power; apartheid; and in 1994 democracy for all (Storbeck et al., 2009). The history of the education of Deaf learners in South Africa was also affected by the country's socio-political 
context for instance with regard to language development and access to education by Deaf learners (Storbeck et al., 2009).

\section{LEGAL AND SOCIO-POLITICAL BACKGROUND}

South Africa has one of the most progressive constitutions in the world (RSA, 1996) protecting the linguistic and cultural rights of Deaf people. Additionally, The South African Schools Act no. 84 of 1996 makes provision for School Governing Bodies (SGB) of schools for Deaf learners to decide if they want to use SASL as the medium of instruction despite the fact that it is not yet an official language: 'A recognized sign language has the status of an official language for the purpose of learning at a public school' (RSA, 1996 (6.4)). This means that SASL in the context of the education of Deaf learners has the equal status of an official language. In other words, SASL and all official languages enjoy parity of esteem and are treated equitably.

However, there is a huge gap between legislation and the reality on the ground at schools for Deaf learners. Linguistic oppression persisted in most schools despite efforts of the Deaf Federation of South Africa (DeafSA). DeafSA is a national federation of associations, groups and schools for the Deaf in South Africa (see www.deafsa.co.za). DeafSA is a member of the World Federation of the Deaf, an international non-government organization of national associations of Deaf people around the world. One of the key objectives of DeafSA is to lobby the Department of Education to introduce SASL as the language of learning and teaching (DeafSA, 2003). DeafSA produced an SASL curriculum in 1996 and started to train Deaf teaching assistants in the same year. This curriculum was presented to the then Department of Education in 1996 but was ignored. In 2003 DeafSA organised a protest march on the Department of Education where a memorandum outlining the linguistic needs of Deaf learners was handed to the then Minister of Education (Morgan, 2008; DeafSA, 2003). This memorandum did not result in any changes in Deaf education language practices in schools. By 2009 (the year of the court case) only 12 of 47 schools for Deaf learners offered matric (Grade 12). Grade 12 is the highest level of achievement learners can reach in South African schools. Moreover, only $14 \%$ of the teachers were fluent in SASL and only a handful of schools chose to use SASL as medium of instruction or were teaching it as a subject (DeafSA, 2009).

In 2009 a court case took place that finally promised the desired changes for linguistic parity. Springate and Others vs The Minister of Education and Others case no. 4846/2009 was instigated by the parents of a matric student, Kyle Springate, who wanted their child to take SASL as a matric school subject and have the opportunity to meet the language entrance requirements for university. They discovered when Kyle was in Grade 12 that SASL was not recognized as a matric subject. The parents were acting as individuals and were represented by the Legal Resources Centre. Interestingly up to that point they had not been involved in DeafSA's fight against linguistic oppression for Deaf education. DeafSA was only brought in later as a friend of the court ${ }^{\mathrm{ii}}$ (DeafSA Education officer, personal communication, 2009).

The case was settled out of court. The settlement consisted of two parts: the first was personal for Kyle since his matric certificate was endorsed by the Minister of Education in order to waive the second language requirement so that he could go to university. The second part of the settlement affected all Deaf students since the 
Department of Basic Education (DBE) agreed to introduce SASL as a subject at schools for Deaf learners up to matric level by 2013. The Department of Basic Education was formed in 1994 when the old Department of Education was split into two departments, namely the Department of Basic Education and the Department of Higher Education and Training. The DBE is responsible for all schools from Grade R to Grade 12 and adult literacy programmes.

This case illustrates the power that ordinary people have in terms of litigation and the seemingly random way in which marginalized groups can achieve their rights. After thirteen years of DeafSA, and its affiliates, lobbying the government, the achievement of linguistic rights and access to education resulted from the brave actions of one Deaf child's parents. The Springate case highlights that Deaf learners were denied the opportunity of studying their first language, SASL, as a subject at school until 2014 when the SASL curriculum was introduced in schools for the Deaf.

After the court case settlement Kyle's mother was quoted as follows in a newspaper article: "The upside is that Kyle has opened the sluice gates. It has been exceedingly difficult for Deaf people in this country to get an education. The majority of Deaf people do not have a matric certificate, so it means they cannot go on to tertiary education." (Broughton, 2009).

\section{Curriculum Management Team}

As a result of the judgement in the Springate case, the Minister of Basic Education appointed a Curriculum Management Task Team (CMT) in 2010. After a call for nominations had been distributed, the DBE selected and appointed the team that would oversee the process of constructing the new SASL curriculum from Grade R12. Grade 12 is the school exit determined level of education in South African schools. This curriculum needed to be implemented by January 2013 so that the first learners ${ }^{\text {iii }}$ would sit their matric or Grade 12 SASL exam in 2016.

The CMT consisted of a heterogeneous group of nine people. Only one of the CMT members appointed was Deaf. Five people including the Deaf member had postgraduate degrees in the field of linguistics and/or Deaf education and had decades of experience working with South African Deaf communities. These five members were the most committed to this process. We knew what was at stake for Deaf learners; as the authors of this paper we knew also that too much time had been wasted and were committed to ensuring that this curriculum development would work. Since we had been fighting for this development/inclusion for over 20 years, it was more than a job.

The other members consisted of two Department of Education officials and a representative from the Pan South African Language Board (PanSALB) whose mandate included promoting and creating conditions for the development and use of SASL. There was also a representative from the Department of Arts and Culture who had been asked to investigate the Parliamentary process of declaring SASL as the $12^{\text {th }}$ official language of South Africa. She stopped attending our meetings during the first year of the process and the CMT never received input regarding this issue. The chairperson was from the Curriculum Directorate of the DBE. Her first teaching job had been at an oral school for Deaf learners; she did not know SASL and had not worked in the field of Deaf education for many years. 


\section{METHODOLOGY}

For this paper we have used a methodology of reflexive deliberation (Archer, 2007) to consider the work of the CMT over a period of four and a half years, from mid-2010 to the end of 2014. Archer (2007) considers reflexive deliberations important as a way of mediating the interplay of structure and agency. As members of the Curriculum Management Team (CMT) established by the Minister of Education to oversee the process of the curriculum writing and rollout, we had an opportunity to shape the process as individuals who were both employed in independent higher education institutions and had experience working in and with Deaf communities for many years. In the process of overseeing the development and implementation of the curriculum we were working within the structural constraints of the State education system.

We have drawn on official documents, our memories of meetings, minutes of meetings where available, official and personal email conversations and text messages between the CMT members where we discussed the current issues, informal text messages and discussions with Deaf people, as well as discussions within the CMT.

\section{Positioning ourselves}

All authors of this paper were nominated onto the CMT by the Deaf communities and individuals with whom they have worked extensively. We were already involved in training, education, research and community development work in the Deaf communities in Cape Town and Johannesburg. Our expertise includes: sign language linguistics, linguistic-anthropology, Deaf education, literacy for Deaf learners and Information and Communication Technology for Development (ICT4D).

\section{Reflexive critique}

It became apparent from the outset that we needed to raise the awareness of the other CMT members regarding Deaf issues around sign language and Deaf culture in relation to education. There were many myths affecting the thinking of the DBE officials. Only one Deaf member had been chosen to be on the team and this was the first issue raised in the initial meeting. We were told that this was not an issue we could discuss as the selection of members was based on qualifications as is common procedure in RSA. Most Deaf people do not have access to tertiary education and therefore could not be considered for selection.

Early discussions were on very basic issues about sign languages, (see Baker et al., 2016). Was SASL in fact a language or not? Even if it was a language, was it sufficiently developed to be used in schools? How do you offer a language as a school subject when it has no written form? These were the first indications that, before our work could begin, much effort and time would need to go into sensitizing and educating DBE officials about SASL and the culture of Deaf communities in South Africa.

One of the early issues was to convince the DBE that we could create an SASL curriculum without the language being fully documented and codified and in the light of there being widespread lexical variation. There was a belief that we could not 
embark on an SASL curriculum before standardizing SASL. The SASL linguists on the CMT argued that, while there is no adequate research on the extent of lexical and syntactic variation or for that matter on SASL phonology, morphology and syntax, this need not be an obstacle to curriculum development. We know that as a result of segregated schooling during apartheid there are often several variants of particular lexical items (Niekerk et al., 2016). All the variants are usually known to native SASL users and lexical variation poses no challenges to communication in SASL, rather it contributes to the richness of the language (Akach and Morgan, 1999).

In addition, the DBE argued that there were no up to date dictionaries or grammars of SASL. There is no textbook yet on the linguistics of SASL. However, we argued that the curriculum could and, would be, taught according to the variety of SASL used by the adult Deaf people in a particular area. Deaf people needed to be employed as teaching assistants and as teachers and Deaf adults in the area around each school needed to be consulted and used as role models for SASL.

The CMT therefore spent the entire first year developing a common understanding and a framework for curriculum development and implementation. In August 2011 the CMT selected the Curriculum Writing Team who were all educators experienced in teaching Deaf learners. Half the team that were nominated were Deaf although one Deaf person never took up the position due to work pressure. She was never replaced despite requests by the Writing Team. The Writing Team were duly appointed and tasked with developing an SASL curriculum from Grades R to 12. Consultants were called in for specific input as needed. The curriculum was completed by July 2013 and tabled in the Government Gazette and published in early 2014 after a process that included amendments as a result of public feedback received.

The process of writing the curriculum over a period of two years went relatively smoothly. The relevant Department of Education (DBE) gave the team autonomy to develop the curriculum and to bring in consultants (educators and linguists) when necessary. Towards the end of the process there was a Deaf stakeholders briefing meeting in Pretoria where the draft curriculum and the proposed implementation plan were well received by the National Chairperson of DeafSA.

The completed curriculum was presented to the Minister of Basic Education in August 2013 where again it was applauded. She requested that the CMT's mandate be extended to oversee implementation and rollout as the DBE lacked the necessary expertise. Unfortunately the inclusive directorate responsible for special schools including schools for Deaf learners was of the view that the curriculum management team (CMT) has completed its mandate and was dismissed. At that momentous meeting we thought that we had successfully negotiated both writing the curriculum and arriving at a common understanding of what needed to happen to implement this new SASL curriculum.

The work of the CMT ground to a halt after the 2013 meeting. Meetings became less frequent. The Chairperson was no longer available at meeting times. Discussions about post provisioning, audit of the schools and materials development were all stalled by the inclusive directorate by not supporting the CMT and arranging its meetings. This culminated in the CMT writing a letter of complaint to the Minister of Basic Education in 2013. To date there has been no response. 


\section{REFLEXIVE DELIBERATIONS}

Our reflexive deliberations focus on the importance of some of the linguistic and cultural issues that emerged as obstacles to the successful rollout of the SASL curriculum. As CMT members we did not have sufficient agency to ensure that these issues were prioritized and structurally accommodated. We are of the opinion that unless these are structurally addressed and resolved they will continue to undermine and jeopardize the success of the rollout. We will conclude with lessons learned during this process.

\section{1) Teaching SASL as a subject vs. as a home language?}

In 2009, the Pietermaritzburg High Court instructed the Minister of Education to introduce SASL as a subject from Grade R to 12. The CMT chairperson explained that SASL could not be offered as a home language as it did not have official language status. However, the CMT pointed out to the DBE that SASL can unproblematically be offered as a home language due to the fact that the South African Schools Act makes provision to consider SASL to be an official language for educational purposes (RSA 1996b). Thus, the curriculum was written for SASL to be taught at home language level and was allocated the increased number of hours allotted to a home language. After much internal discussion the final decision was to offer it as a subject at home language level.

The issue of whether SASL is offered as a home language or not is complex. To offer it as a home language ensures that it has first language status for Deaf learners and that English can then be offered as a first additional language. Although for most Deaf learners SASL is not used at home due to the reluctance of family members to acknowledge and use SASL, it is the language that Deaf learners acquire from other Deaf learners and adults at school. It is the language to which they have full access making it the easiest to acquire and is the one in which they are the most proficient.

\section{2) Bilingual-Bicultural team teaching}

There is a lack of qualified Deaf teachers to teach SASL as a result of barriers to tertiary education and teacher training for Deaf South Africans. In the absence of a qualified Deaf teacher, the CMT proposed a bilingual-bicultural team teaching model following Akach (2010) in which a hearing teacher would be paired with a Deaf teaching assistant. The most important reasons being both cultural and linguistic. SASL does not have high status in schools for Deaf learners where English is promoted as the language necessary for success in education (Nkwinika, 2013). It is therefore important to provide proficient Deaf SASL role models to Deaf learners. For cultural reasons, it is imperative that no hearing teachers teach SASL alone. The reality on the ground is that most hearing teachers are not native sign language users, and do not have the linguistic proficiency to teach SASL.

The situation regarding Deaf teachers and Deaf teaching assistants using SASL to teach SASL is also complicated. Being Deaf and a qualified teacher does not necessarily ensure that teachers are fully proficient in SASL nor that they use SASL in the classroom as the language of learning and teaching. Nkwinika (2013) found that two of three Deaf teachers who claimed to be using SASL as medium of 
instruction in a school that promoted bilingual education were in fact using different combinations of features of SASL and English. They would use SASL word order with English mouth patterns or signs presented in English word order with English mouth patterns. In both of these examples no non-manual signals are used. Any of these combinations results in the degradation of both languages (Schwartz 1996). This situation was also present in another school that claimed to be using SASL as medium of instruction. The SASL materials they provided included Afrikaans mouth movements accompanying SASL signs.

According to Nkwinika (2013) the reasons given for not using SASL were that it had not been prioritized in schools for Deaf learners. Deaf teachers had been mainstreamed during their own schooling and had internalized English as the more important language. They were most comfortable signing a mixture of English and SASL and moving between the Deaf and hearing worlds. They therefore had bilingual identities. The only teacher in this study who signed SASL proficiently had been to a residential school and had a strong Deaf identity.

The reality in many classrooms in schools for Deaf learners is that Deaf and hard-ofhearing learners are taught together. This puts an unrealistic demand on teachers to provide input in English and SASL at the same time; a linguistically impossible feat. Teachers usually resort to a mixture of SASL and English as described above. More research needs to be undertaken in the area of Deaf teacher proficiency in SASL and their cultural identities.

For implementing the SASL curriculum we need teachers with both teaching skills and SASL skills. The CMT suggested that this is only possible with qualified Deaf teachers proficient in SASL. Where this is not possible, the CMT advocated team teaching.

The training implications for Deaf-hearing team teaching involve emphasizing the importance of Deaf teachers and teaching assistants using SASL structure as it is used in the Deaf community by proficient SASL users. The status of SASL needs to be raised among teachers. Teachers need to be trained to distinguish between SASL, Sign-supported English and English-supported SASL and to get their learners to do the same. Akach (pers. comm. 2014) informed us that based on his experience working with Deaf and hearing teachers and assistants, Deaf teaching assistants need to first be empowered to acknowledge and be proud of their expertise in SASL. There is a history of oppression of Deaf teaching assistants and they have internalized feelings of lack of worth and believe that SASL has less status than English. There is also a history of hearing teachers getting Deaf assistants to do menial non-teaching related tasks. The Deaf assistants are often working in those same schools that failed them as pupils. The situation is delicate and Deaf and hearing teachers and assistants have to learn to relate to each other as equals in order to work in a team where the Deaf assistant has expert knowledge of SASL and the hearing teacher has expert knowledge in terms of teaching methodologies, classroom practice and following a curriculum that has been written in English. Although the CMT recommended that the curriculum be recorded in SASL for Deaf teachers and assistants, this has not been done.

The main factor determining success of using team teaching in the implementation of 
the curriculum would be the ability of the Deaf and hearing members of the team to trust and respect each other. Both Deaf and hearing teachers and Deaf teaching assistants need training in areas such as sign language linguistics, socio-linguistic variation, poetry, storytelling, discourse analysis and Deaf culture. Furthermore, the Deaf teaching assistants need opportunities to become certified teachers.

\section{3) Implementing the rollout}

Despite the court requirement for the rollout to start in January 2013, the DBE set the due date for implementation for January 2014 due to systemic delays. However, given these delays, it became apparent to the CMT that the system would not be adequately prepared for rollout by then. We therefore requested that 2014 be a year of preparing the system for rollout in 2015. This was to ensure that implementation was not rushed and that it would properly meet the needs of learners.

We needed to ensure that additional post provisioning for Deaf teaching assistants was fast tracked through the system, so they could be filled before staff training started in July 2014. This was when an initial introduction to teacher training had been planned. Appropriate teaching guides and SASL learning and teaching support materials (LTSM) needed to be developed. Each school needed to be resourced with the appropriate computer, filming and video equipment.

By the end of 2013 the majority of the CMT members became aware that we were experiencing serious challenges in terms of leadership, and financial resources. Our chairperson had stopped attending meetings after the presentation to the Minister of Education. There were many challenges that needed to be resolved at the beginning of 2014.

A critical issue was who would drive this rollout process at national, provincial and district level. The CMT had been previously informed by the chairperson before the presentation to the Minister of Education that a new office at the DBE could be created and staffed to oversee the SASL implementation process. She also suggested we set up a national training, monitoring and evaluation unit at one of the universities and offered to contact one of the Vice Chancellors. We had decided that ongoing training and support at every school were necessary.

As outsiders we had no authority to implement but could only provide recommendations and guidance. All actions had to be carried out by DBE officials following internal procedures. There was little flexibility to accommodate the specific needs of teaching SASL. At the end of 2013 we were informed that there were no more funds available to plan any further meetings, events or training in early 2014 until the DBE received its budget allocation after the start of the new financial year. This meant that funds would not be available until midyear 2014. On our insistence two planned events took place early in the year: a meeting with Deaf stakeholders and an orientation for senior management teams at schools. Teacher training needed to be postponed until July 2014.

At this time we became increasingly concerned regarding the possibility of the system being ready by January 2015 for the planned rollout. We drafted a submission to the Minister of Education outlining these concerns. 
As 2014 was an election year, nothing happened at the DBE between mid February and the end of March. In April the CMT's submission to the Minister of Education was dispatched. We were advised that this submission should not go directly to the Minister but through the ranks of the DBE, so that all senior officials would be informed and briefed before she received it. The main thrust of the submission was to outline the leadership, financial and other problems that we had experienced. Our bottom line was that, unless new posts for Deaf teaching assistants were fast tracked and created, we could not go ahead with the teacher training planned for July 2014.

During an inventory carried out by the CMT in the previous year of all schools that wanted to implement the SASL curriculum one of the main issues that emerged was the lack of sufficient Deaf teaching assistants. For this curriculum to be implemented correctly in the absence of a Deaf teacher, each class needed both a Deaf teaching assistant and a hearing teacher. For Foundation phase there were often two classes per grade. This meant that ideally each school should have eight foundation level assistants and at least one more for Grade 9.

We never received a response to our submission. In July 2014 a CMT member emailed asking for information. The response was a very short email saying that the CMT had fulfilled its mandate and were no longer needed. The authors were horrified and shocked. We felt that we were being disbanded in order to be silenced so that the DBE could go ahead and do training without making any costly structural changes. We wrote letters to the Director of the DBE which he neither acknowledged nor replied to. Central to our outrage was our feeling of responsibility. We were accountable to the Deaf communities who had nominated us onto the CMT. Despite our dismissal we remained the contact points for Deaf organizations and individuals who were relying on us to drive the process.

The DBE conducted teacher training in October 2014 using mainly internal staff due to lack of funding to include outside trainers, including the CMT. This training team included only hearing people, none of whom have near native fluency in SASL. The DBE then included two of the CMT members (one of whom is Deaf and the other a skilled interpreter with near native proficiency in SASL) to participate in the training. One of the CMT members was unsure whether to get involved after being summarily dismissed or to stand firm on the grounds that the Deaf teaching assistants had not yet been appointed. After email discussions we finally felt that it would be worse for Deaf learners if two of our members were not involved since the DBE was going ahead with the implementation regardless of what concerned members of the CMT did. Two CMT members decided to try to salvage the quality of the training. As one of the CMT members was never given a letter of invitation or any documentation as a trainer he only completed one week of the training. The Deaf CMT member agreed to do the training out of his passion and commitment to Deaf learners.

As an interim measure the DBE decided to empower language subject advisors with no knowledge or experience of SASL to provide monitoring and support to schools for Deaf learners that would be implementing the curriculum in 2015. This was done in a two-day orientation session. They would remain responsible until SASL subject advisors had been appointed in each province.

The CMT had initially proposed a three-week training period for Deaf and hearing 
teachers and assistants to be followed by ongoing support and training at their actual schools. The actual training lasted in fact only two weeks.

Feedback in the form of text messages to a CMT member from Deaf participants revealed confusion and frustration concerning several instances of incorrect linguistic information. For example, according to the native language intuition of Deaf first language SASL users, the word order of SASL presented and prescribed by a hearing educator was incorrect. A cursory review of the phonological parameters section of the training materials revealed an incorrect understanding of the notion of non-manual features. Firstly the idea that non-manual features are optional is incorrect as they are grammatically required (Pfau \& Quer, 2010). Furthermore, the corresponding table with illustrations and examples for each parameter is also incorrect. Those in the nonmanual features column are in fact referring to affective facial expressions, e.g. cross, delicious, concentrate, amaze, delicious and unbelieving. They included grammatical markers such as yes-no questions and questions in this category but they are undifferentiated from the affective facial expressions. It is extremely concerning that there is no mention of other non-manual syntactic markers such as topicalisation, negation, affirmation and adjectival and adverbial markers such as intensifiers (Pfau \& Quer, 2010).

The CMT ensured that the correct linguistic information was included in the curriculum by calling in additional sign language and linguistic experts into the writing process. The fact that neither the CMT nor any linguistic experts were involved in the preparation of the training materials resulted in teachers not being able to understand the linguistic content of the curriculum. Furthermore, some of the Learning, teaching and support materials (LTSM) submitted to the team responsible for the compilation of the SASL LTSM National Catalogue were rejected on the grounds that they did not conform to the contents of the training materials although they were in line with the curriculum. One of the authors received a query regarding the nature of classifier handshapes from an organization that submitted LTSM SASL stories for the Foundation phase for review. Some of their SASL materials were rejected by the DBE on the basis of the use of a single classifier handshape which was deemed not to conform to their accepted set of handshapes for animals in SASL. As there is very little research on SASL classifiers, it is hard to imagine where this definitive set of classifier handshapes came from. In addition, one of the significant characteristics of classifiers in sign languages is their productive and creative nature, especially in the context of literature which in SASL includes storytelling (SuttonSpence and Kaneko, 2016). Much time and effort had to be expended before the DBE accepted these linguistic facts.

There was also dissatisfaction from the participants about the power relations between Deaf and hearing people. Development of the training materials and the actual training was led by hearing people. Although one Deaf member of the CMT and another hearing person with near-native SASL proficiency were involved in the training, they were co-opted at the last minute. They were not able to provide input into the content of the training despite the fact that they are highly qualified educators with extensive experience in the field. The two CMT members were already allocated topics to present to the teachers and were informed by the chief trainer that powerpoint presentations were prepared and ready for them to use. The two members were told that what was required was to study the slides and present them. However, 
preparation time was not sufficient and the members had to rely on their knowledge of the topics. This became difficult when the information on the slides and the members' knowledge of the topics did not match. The training was therefore inadequate in terms of the level of the linguistic skills and cultural sensitivity of the people responsible for developing the training materials, the quality of the linguistic content of the training materials and the time allocated for training.

We interpret these events as the result of inadequate linguistic knowledge and cultural sensitivity within the DBE and that this underlies the breakdown of the process. The process was ultimately structurally constrained due to decisions made at a directorate level in the DBE. By dismissing the CMT the DBE were excluding a team that collectively had the required linguistic knowledge and sensitivity.

\section{LESSONS LEARNT}

This process has made important aspects clear with regard to the relevance of linguistic knowledge and cultural sensitivity of all individuals involved at all levels in creating and implementing the SASL curriculum.

The process of writing the SASL curriculum went relatively smoothly and a quality product was produced as assessed by the public comments received before the final approval and publication of the curriculum. This success can be attributed to the Writing Team having the necessary skills for the task. It was a mixed team of Deaf and hearing members. They were all educators with experience teaching Deaf learners and were able to consult linguists and educational experts as necessary for input. The curriculum is culturally sensitive to Deaf learners' needs and allows for linguistic variation. It is not prescriptive and is constructed according to what is currently known about the linguistic structure of sign languages and provides space for Deaf teachers to use the variety of SASL that is used by the surrounding adult Deaf community. The CMT oversaw the process and some members of the CMT as well as other specialists were invited to be involved in the writing sessions as needed. The agency of both the individuals in the Writing Team and the individuals on the CMT was not questioned by anyone higher up in the hierarchy of the DBE we were working within.

It was only when the CMT demanded that the Deaf teaching assistants be appointed before training commenced, that we were summarily disbanded. The structural constraints within which we were working overrode our agency as a team with the majority of members strongly opposing decisions that were being made higher up in the hierarchy of the DBE at Directorate level. We argue that without the CMT's linguistic expertise and cultural sensitivity the early implementation to get the system ready for rollout in 2015 was not effective. The initial two weeks of teacher training was problematic in terms of the inadequate preparation of the trainers, the inadequate SASL skills and linguistic knowledge of the trainers resulting in problems with the content of the training materials and the time allocated for training.

Perhaps the rush to get the curriculum into schools without trying to meet the linguistic and cultural needs of Deaf educators and Deaf learners resulted from a desire to avoid being in contempt of court. There was a court settlement that needed to be adhered to and the DBE was already at least two years late according to the 
original time frame. It appears that the DBE simply wanted to follow court orders without the required budget to do this adequately and deliver the SASL curriculum by 2015 without any further delays, regardless of concern for quality of the rollout.

The Training Team that was responsible for the training of teachers on a national level after our departure is confident that implementation will succeed in some of the schools. However, the authors argue that the success of this process would be best evaluated in those under-resourced schools and not the few resourced schools that already have established SASL as a subject prior to this process. In the underresourced schools there are fewer Deaf teaching assistants, fewer opportunities for SASL training, limited computer equipment and learning and teaching materials and the reliance on language subject advisors who have had no exposure to SASL. The majority of schools sorely need ongoing training, materials and structural support from people accepted and respected by Deaf communities, with SASL proficiency, linguistic expertise and cultural sensitivity honed from years of collaboration with these communities. It is hard to imagine that the DBE would implement a rollout of a new curriculum for any other language under these circumstances. This suggests strongly an underlying absence of respect for the integrity of SASL.

An important lesson that emerges from the teacher training and materials development is that it is dangerous to follow a prescriptive approach regarding the structure of SASL and the assessment of SASL materials for use in the classroom. Due to the extent of variation on all linguistic levels (lexical, morphological and grammatical) teachers need to use whatever is used by the adult signers in their communities. They need to be taught the principles of sociolinguistic variation and accept the rich diversity of SASL signs and structures.

A serious concern that emerged in the training was the way the issue of lexical variation was dealt with, specifically in relation to the standardization of terminology. While the DBE's National Training Team asserts that all dialects will be accepted and respected, they advocate that for the purposes of teaching and materials in schools a standard sign should be used, for example that a single sign should be used in all schools for subject specific terminology such as PHONOLOGY. However, there was no consideration of how this could possibly be achieved, that is who decides which sign should be used and how this could be policed. This again reveals a misconception of how languages develop and how terminology evolves by users in a natural context.

Teachers and learners need to be encouraged to claim their own agency as researchers in the classroom. There is insufficient research on the structure of SASL - we need to document this language together and teach in a way that is descriptive. Sign language variation needs to be understood and accepted. We need to find out what is being done by learners as well as adult Deaf people at the local level, create partnerships between Deaf communities and schools and provide appropriate teacher training in different areas of SASL structure and use.

The fact that a lack of financial resources interfered with the implementation process also needs to be addressed. The issue of funding should not just be used as a reason to deliver an ill-conceived training programme. If funds are not available within the DBE, there needs to be a partnership with a resourced educational organization or a 
fundraiser appointed to raise the necessary funding for training and ongoing mentoring, monitoring and evaluation.

To take this process forward we need to have the resources as well as more people, especially Deaf people involved in training, materials development and ongoing teacher mentoring who are knowledgeable about SASL linguistics and Deaf cultural identity as well as with experience in teaching SASL.

Another problem was the decision from the DBE to follow the court order that SASL be taught as a subject rather than as a home language despite legal provision in the Schools Act. Although the Curriculum for SASL is written at home language level, this decision accords SASL less status than a home language would have thus sending out a negative message to Deaf learners. This is exacerbated by undertrained hearing teachers with uncertified Deaf teaching assistants using hastily collated materials and insufficient support from language advisors at the district level as there are very few, if any, skilled SASL educators working in the district offices.

\section{CONCLUSION}

Out of our reflections, according to Archer's (2007) notions of the interplay between agency and structure, emerges the realization that structural constraints and limitations dominated over individual agency in the end. After the removal of the CMT, there was a reorganization of the oversight of the process of implementation that excluded us. The four of us who were the most invested in this process on behalf of Deaf learners considered our options - The national chairperson of DeafSA wrote to the Minister of Education voicing the complaints of the Deaf community. One of our team members wrote on behalf of the CMT to the Minister of Education for redress. The Minister has remained silent. It is nearly two years later now.

The main lesson is that the implementation needed visionary leadership at a structural level within the DBE. Without the agency of a leader within the DBE structure, the quality of the rollout of the curriculum was severely compromised. In order for agency to have a chance in relation to structural control, the individual responsible for driving this process at a high level within the DBE needed to have the time to devote to this process. It required being passionate about the rights of Deaf learners to study SASL as a home language at school from a trained Deaf person. S/he also needed to be aware of the importance of knowledge about SASL, Deaf identity and Deaf culture informing the training, materials development and rollout. This individual should embody the core value of respecting the basic linguistic and cultural rights for Deaf South Africans enshrined in our Constitution, namely the right to quality education, the right to dignity, equality, the right to receive and impart information, to access information and the right to use the language of your choice, in this case, SASL (RSA, 1996a). Education is essential for further skills development for employment and affording Deaf people their right to vote and full participation and inclusion in society, as citizens of South Africa. 
${ }^{\mathrm{i}}$ The use of capital D follows the convention of distinguishing "a signed-language using community of people who do not hear (Deaf people) from non-signing people who do not hear (deaf people)" (Humphries, 2013: 1).

ii An organization with a specific focus gets leave from the court to assist the court to give input on the important social-economic or similar issues. In this case their input assisted in understanding issues pertaining to discrimination experienced by Deaf people in relation to sign language and education despite their constitutional entitlement to language, education and non-discrimination.

iii The new curriculum production was given a year with a view to implementation occurring in 2011 and 2012 for foundation phase and Further Education and Training (FET) to ensure the first SASL matriculants in 2013.

\section{REFERENCES}

AKACH, P. 2010. Application of South African Sign Language (SASL) in a BilingualBicultural Approach in Education of the Deaf. PhD. Dissertation submitted to the University of the Free State.

AKACH, P \& R MORGAN. 1999. Community Interpreting: Sign Language Interpreting in South Africa. In An introduction to liaison interpreting. Edited by Mabel Erasmus, Hugo Antonissen and Erik Hertzog. J L Van Schaik.

AKACH, P. 2014. Personal email communication.

ARCHER, M. 2007. Making our way through the world: Human reflexivity and social mobility. Cambridge: Cambridge University Press.

BAKER, A, B VAN DEN BOGAERDE, R PFAU \& T SCHERMER. 2016. The Linguistics of Sign Languages: An introduction. John Benjamins.

BREW, A, D BOUD, L LUCAS \& K CRAWFORD. 2013. Higher Education. DOI $10.1007 / \mathrm{s} 10734-012-9592-6$.

BROUGHTON, T. 2009. The Mercury. 20 August 2009. Independent Newspapers.

DEAFSA (Deaf Federation of South Africa). 2003. Memorandum to the National and Provincial Departments of Education. 7 February 2003: Johannesburg.

DEAFSA (Deaf Federation of South Africa). 2009. Deaf learners and their education rights: Is South Africa listening? Available from www.deafsa.org.za. [Accessed: 19 January 2011]

DEAFSA (Deaf Federation of South Africa) Education officer. 2009. Personal email communication.

HUMPHRIES, T. 2013. Schooling in American Sign Language: A paradigm shift from a deficit model to a bilingual model in deaf education. San Diego: University of California.

MORGAN, R. 2008. Deaf me normal: Deaf South Africans tell their life stories. Hidden Histories Series. Pretoria: Unisa Press.

NIEKERK, A, D EBERSON, K HUDDLESTONE \& A BAKER. 2016. Charting Lexical Variety in SASL. Presentation to the LSSA meeting, Cape Town, July 2016.

NKWINIKA, N. 2013. How Deaf teachers use South African Sign Language (SASL). Honours Research Essay. SASL Department. University of the Witwatersrand. 
PFAU, R \& J QUER. 2010. Nonmanuals: their grammatical and prosodic roles. In Diane Brentari (ed.) Sign Languages, Cambridge: Cambridge University Press. 381-402.

REPUBLIC OF SOUTH AFRICA. 1996a. Republic of the Constitution of South Africa Act, no. 108 of 1996. Pretoria: Government Printer.

REPUBLIC OF SOUTH AFRICA. 1996b. South African Schools Act, no. 84 of 1996. Pretoria: Government Printer.

SCHWARTZ, S. (Ed). 1996. Choices in Deafness: A Parents' Guide to Communication Options. Woodbine House. $2^{\text {nd }}$ edition

STORBECK, C, L MAGONGWA, \& I PARKIN. 2009. Education of the Deaf in South Africa. In DF Moores, and MS Miller, (Eds), Deaf People around the World: Educational and Social Perspectives. Gallaudet University Press: Washington, $D C$.

SUTTON-SPENCE, R \& M KANEKO. 2016. Introducing Sign Language Literature: Creativity and Folklore. Basingstoke: Palgrave Macmillan

\section{BIOGRAPHICAL NOTES}

Ruth Morgan lectures in the South African Sign Language Department, School of Literature, Language and Media, Wits University. She did her postgraduate studies in the USA including an MA in linguistics from Gallaudet University and a $\mathrm{PhD}$ in linguistic anthropology from The American University. She has worked in the field of Deaf cultural studies and sign language research since the late 80s.

Meryl Glaser is an honorary Research Fellow in the School of Literature, Language and Media, Wits University. She is a facilitator and researcher consulting on Deaf issues in education, language, and literacy. She has an M.Sc. in Human Communication (specializing in Deaf people) from the City University, London. She collaborates on various ICT4D projects with the Computer Science department at UCT.

Lucas Magongwa is a lecturer and coordinator of Deaf Education courses in the Wits School of Education at the University of the Witwatersrand, Johannesburg. He teaches in the areas of South African Sign Language (SASL) and Deaf pedagogy. His research interests are SASL teaching and learning, Deaf teacher development and developing teaching practices that promote the education of Deaf students. Lucas is currently a $\mathrm{PhD}$ candidate. 